\title{
Systems approaches for pharmacogenetics and pharmacogenomics
}

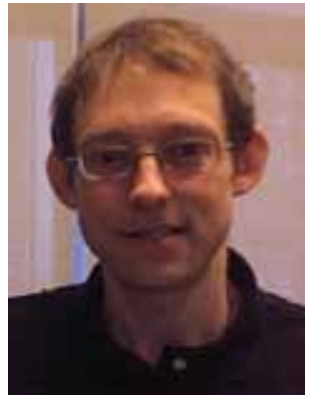

Jœ S Bader

D epartment of Biomedical Engineering and $\mathrm{H}$ igh-T hroughput Biology Center, Johns H opkins University, 201C Clark H all, $3400 \mathrm{~N}$. Charles Street Baltimore, M D 21218, U SA Tel.: +1 410516 7417; E-mail: joel.bader@jhu.edu

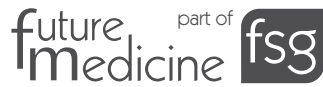

\author{
'Ra ther than linking a \\ phenotype to a single gene, the \\ new paradigm may be linking a \\ trait to a pathway or a \\ biologica I system.'
}

The search for genetic variants that influence medically-relevant phenotypes has been driven by the central dogma of genetics: DNA encodes genes as discrete units of inheritance; genes encoded by DNA are transcribed to RN A; messenger RN A molecules are translated to proteins; one protein has one function; and aberrant proteins encoded by mutant gene variants lead to aberrant biological activity. This paradigm explains many human diseases [1] and has been extended to diseases where two or perhaps three genes contribute [2].

Unfortunately, the paradigm of 'one gene, one protein, one function' has failed to reveal the causes of prevalent complex diseases known conclusively from twin studies to have a genetic basis [3,4]. These diseases include arthritis, diabetes and risk factors for cardiovascular disease. Similarly, this paradigm may also fail for traits related to pharmacokinetics and pharmacodynamics.

Technological advances in genetic analysis now permit whole-genome association, in which the entire genome of an individual is screened for genetic variation at a series of preselected locations, termed markers. In these studies, there are approximately as many markers as individual genes, if not more. SN Ps are the default choice for markers, and SNP sets for the human genome now have $100,000-500,000$ markers to provide coverage of our 24,000 genes. Testing these markers for association with a phenotype is a challenging signal-to-noise problem: it is difficult to distinguish between the few real causative effects and the statistical flukes that are expected from examining 100,000 markers, whether markers are individual SN Ps or correlated local patterns of SN Ps termed haplotypes [5].

It is now possible to test hypotheses that phenotypes result from cumulative, subtle regulatory changes, in contrast to the catastrophic failure of a single protein as in classic $M$ endelian genetics (Figre1). Rather than linking a phenotype to a single gene, the new paradigm may be linking a trait to a pathway or a biological system.

Although linking genetics to pathways is an intriguing idea, the lack of systematic pathway maps beyond basic metabolism has presented an insurmountable obstacle. Consequently, most work in human genetics turned to other avenues; the theoretical framework for pathway-level mapping is an important current avenue of research. Only recently, in part owing to the author's work [6], have pathway maps become available to the extent that they can be used in genetic analysis.

\section{Overview of the challenges}

A schematic illustrates the challenges in understanding genetics data for a complex phenotype such as response to a drug (Figure2). In this picture, the quantitative level of response corresponds to the quantitative level of activity of a biological network. Genetics can identify which pathway is involved, but requires several steps of inference, each with its own challenges:

- From a disease to a genetic marker (wholegenome association): identify a physical region of the genome that houses a genetic variant that affects the trait value. Challenges: regions with small but real effects (expected for complex traits) are easy to miss. Variants that interact synergistically are even more difficult to detect.

- From a genetic marker to a gene (candidate gene identification): identify the gene or genetic variant within the region that is responsible for the effect. Challenges: regions may contain several genes, making it difficult to conclusively point to the gene responsible for an effect.

- From candidate gene to system (systems biology): understand the systems-level architecture of a disease by mapping causative genes back to biological pathways. Challenges: this step lays inference upon inference because mapping markers to genes is already difficult. 
Phenotype to marker: whole-genome association

- G enetic association tests are designed to identify correlations between genetic variations and phenotypes, which may either be discrete classes such as case versus control, or quantitative measures such as parameters in a pharmacokinetics/pharmacodynamics model. A marker that is associated with a phenotype might not cause the phenotype of interest. Instead, the marker might be located physically close to the functional variant so that the marker allele is tightly correlated with the functional allele (Figre3). The precise meaning of 'physically close' depends on the type of study. In family studies, correlations depend on the typical distance between meiotic recombination sites, roughly $50-100 \mathrm{M} \mathrm{b}$. In a population of unrelated humans, allelic correlations decay much faster, with a length scale of $10-100 \mathrm{~kb}$. This linked region for a quantitative trait is termed a quantitative trait locus $(\mathrm{QTL})$. Since the human genome has approximately 3 billion nucleotides, the number of markers required is of the order of 10,000-500,000.

\section{Figure 1. Functional effects of DNA variants in protein-coding and regulatory regions.}

A

Wild-type

М00000000000000
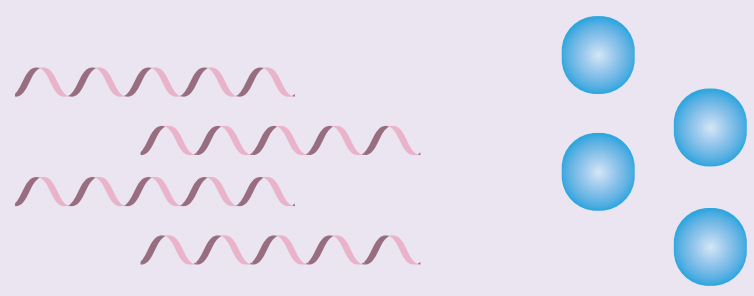

B

Protein variant
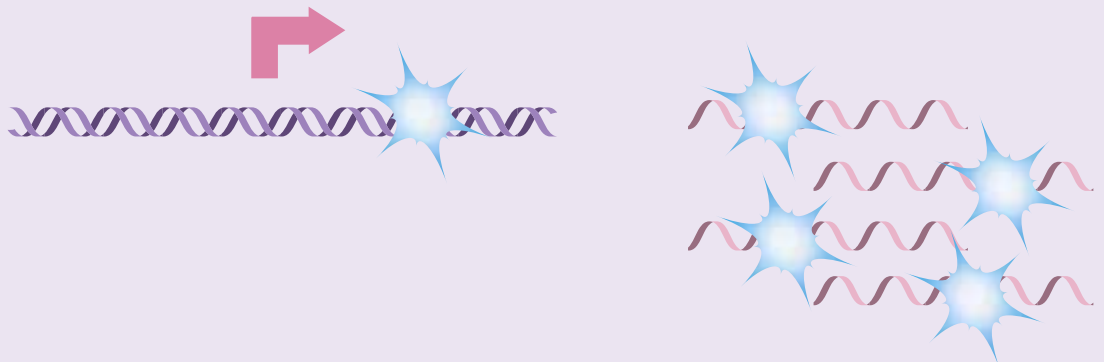

C Regulatory variant
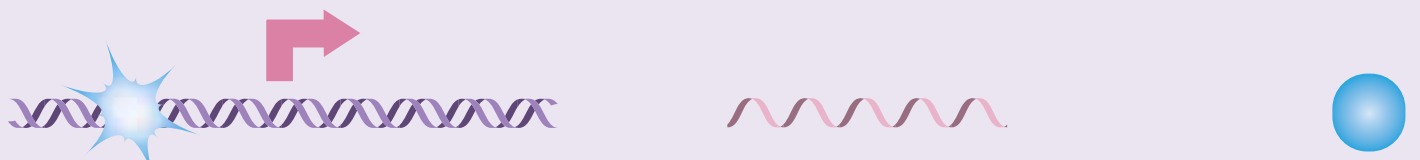

DNA

mRNA

Protein

(A) The central dogma of genetics is that DNA encodes mRNA, mRNA encodes protein, and proteins have discrete functions. Genetic diseases arise when mutant DNA encodes a protein with aberrant function (B). Disease may also arise when proteins in a pathway are not properly regulated, even though the proteins themselves may be perfectly normal (C). These changes can be due to variation in regulatory DNA that does not encode protein. Organizing genetic analysis using systems-level knowledge will identify disease-causing variants. 
Figure 2. Identifying the system of genes responsible for a phenotype requires backwards inference from phenotypes to markers, from markers to genes and from genes to pathways.

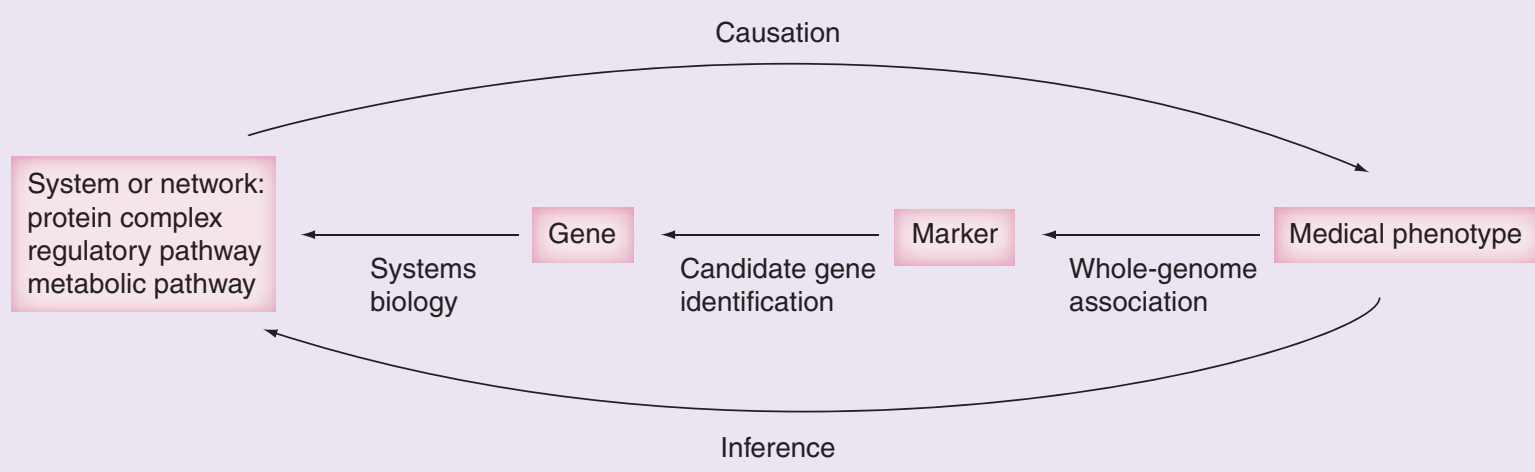

When conducting a study with upwards of 1 million markers, it is important to reject false positives owing to statistical flukes that are expected to occur at roughly the one-in-a-million level, or a $p$-value of $10^{-6}$ or smaller. Establishing a real finding at this significance level for a complex trait can require studies with thousands of individuals (Figre3). Costs for enrolling each individual in a study and performing genotyping are of the order of U $\$ 1000$ or more, for a final cost of millions of dollars. Projects like the $\mathrm{H}$ apM ap aim to optimize the markers used [7], but do not deviate from these basic economics.

At costs of U S\$1 million a study, it is essential to understand how different methods perform before applying them to human populations. Realistic assessments require the use of model organisms. Yeast (Saccharomyces cerevisiae) is perhaps the single most widely used genetic model organism: it is the simplest eukaryote that is widely studied; it was the first eukaryote to have its genome sequenced; and it has a panoply of genetic tools. These tools include the ability to knock out or perturb gene function [8], which is important for verifying results of genetics studies; widely available transcript profiling techniques to measure the activity of every gene at the mRN A level; and the most advanced proteomics methods to map the composition of protein complexes and pathways $[9,10]$.

Yeast alleles and phenotypes vary between strains. By mating yeast cells from different strains, collecting the progeny, scoring the daughter cells for a phenotype and then genotyping the same cells, it is possible to identify genetic regions or loci that are associated with the same phenotype. In a well-controlled experiment, this association establishes a causal linkage between a specific DNA region and a trait. When traits are themsel ves the output of a high-throughput experiment, this strategy is termed 'genetical genomics' [11].

These types of experiments have now been performed for a range of yeast phenotypes related to human disease: drug response [12], telomere maintenance relevant to cancer and aging [13]; and aberrant cellular shape or size [14].

'At costs of US\$1 million a study, it is essential to understand how different methods perform before a pplying them to human populations.'

An influential series of experiments used gene regulation as a trait, with roughly 6000 phenotypes measured in parallel, each phenotype corresponding to the mRNA abundance of each yeast gene $[15,16]$. The DNA region causally linked to an mRNA is termed an expression quantitative trait locus ( $\mathrm{QQTL}$ ). Since variation in gene regulation contributes to complex disorders (Figre 1), mapping eQ T Ls is very relevant to human disease [17]. M apping eQTLs in model organisms has identified DN A regions relevant to obesity [18] and other disorders [19].

A method successfully used to improve statistical association has been dimensional reduction. Traits that are highly correlated, for example, genes whose expression levels track each other closely, can be tested as a batch. This reduces the multiple-testing correction. Standard statistical techniques, such as principal components analysis $[18,20]$ and clustering $[17,21,22]$, have been used to reduce the number of tests. 


\section{Figure 3. The mathematics of association tests.}

Assocation test $\longleftrightarrow$ Regression test for non-zero slope

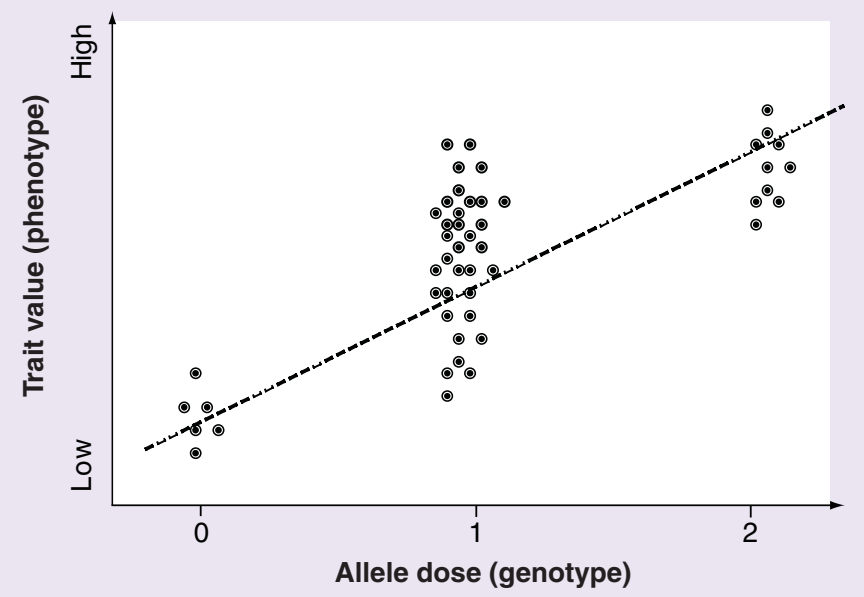

An association test for a complex quantitative trait can be performed as a regression test of trait value against allele dose (number of copies of a particular allele for a specific marker). The fraction of variance explained by the gene is denoted as $R^{2}$, corresponding to a regression line with correlation $R$. A simple equation relates the effect size, the number $(n)$ of individuals genotyped, and the expected statistical significance: $z^{2}=N R^{2}$, where $z$ is the number of standard deviations away from the null expectation for a 50:50 chance of detection. A p-value of $10^{-6}$ corresponds to 4.8 standard deviations, and $\mathrm{R}^{2}$ for a complex trait can be $1 \%$ or lower. For this typical example, 2300 individuals are required just to have a $50 \%$ chance of a significant finding.

Spurious associations may arise from the technologies used to measure transcript levels. The most common method used to measure transcript levels is hybridization to wild-type sequences on a chip. If a D N A variant hybridizes less efficiently, that gene will appear to be underexpressed, even when it is present at normal levels. These variants can be correlated with nearby variants used for markers, leading to a false-positive association. This problem has been recognized [23,24]. Allele-specific expression can be used to distinguish between real cisregulatory variation and a spurious effect $[25,26]$.

Most association studies focus on main effects of a single variant, equivalent to a statistical test for correlation between trait value (gene-expression level for an eQTL) and genotype at a single marker. A standard statistical technique to identify additional markers is to subtract the effect of a marker, then identify other markers that may have smaller effects. This is equivalent to a forward regression model based on partial correlation coefficients. An additional complication is caused by epistasis, in which the effects of multiple variants combine nonlinearly. This is equivalent to adding interaction terms to a multiple regression model. M ultimarker tests have been difficult to apply in practice because, with 6000 markers as in the yeast studies, each additional marker requires an additional 6000 -fold correction to a p-value. H uman studies, with 100,000 markers or more, would have more difficulty to find statistical significance for any multimarker test. Calculations must also be organized for efficiency. Thus, only a few multimarker tests have been described, and to date only for yeast $[27,28]$. These studies have shown that multimarker effects are important, with approximately two-thirds of markers missed based on main-effect analysis al one. Furthermore, approximately half of the two-marker models have shown significant nonlinear interaction terms. Improving multimarker tests beyond brute-force methods will be necessary to identify multigene effects in humans.

\section{Marker to gene: candidate} gene identification Identifying a physical segment of D N A linked to a trait is only the start of identifying the causative variant. Indeed, almost all of the studies cited above stop at the level of marker association, without ever identifying the genetic variant or even the gene responsible. I dentifying and validating candidate genes is an outstanding challenge [29]. For $\mathrm{eQTLS}$, two types of linkage are generally described, as described below.

'Research is now needed to develop algorithms that integrate whole-genome associa tion stud ies with growing knowledge of networks and pathways.'

ciseQ TLs are markers that are physically close to the gene being regulated. The causative variant could be nucleotide substitution or insertion/deletion in the promoter region of the regulated gene that affects transcription factor or polymerase binding. ciseQ T Ls may also arise from changes in introns or untranslated regions (UTRS) in the RNA transcript that flank the protein-coding sequence that affect RN A processing or stability. In most cases the marker is not the causative polymorphism, which remains unknown.

trans $\mathrm{eQ} T \mathrm{~L}$ s are markers that are far from the regulated gene. Suppose that gene $A$ regulates the expression level of gene B. A mutant copy of 
A that produces a protein with aberrant function could result in misregulation of gene $B$. A more subtle effect arises if the sequence of protein $A$ is correct, but a variant upstream of gene A causes a change in its expression level that in turn affects the regulation of gene $B$. In this case, a cis-eQ $T L$ near gene $A$ is al so observed to be a trans-eQ TL for gene $B$. It is difficult to go from a marker to a gene, however, because a trans-eQTL can be linked to several adjacent genes, with no statistical evidence to support one candidate over the others.

An intuitive explanation for a trans $\mathrm{eQT} \mathrm{L}$ is that it encodes a transcription factor, the class of protein most directly related to gene regulation. Unfortunately, investigations of $\mathrm{eQTL}$ regions have yet to show any enrichment for transcription factors. Nonsynonymous changes to protein-coding regions are another class of probable causative variants [30], but probably represent a minority of trans-eQ T Ls.

The method that has so far had the greatest success for trans-eQTLs is to combine genetic association with gene-expression correlation: look for a candidate gene that is close to an $\mathrm{QQTL}$ for a particular transcript and whose expression level correlates with that same gene [31-34]. This type of integrated analysis is promising.

\section{Gene to pathways: systems biology} Systems biology is an integrative science that attempts to understand gene function in the context of larger biological units: protein complexes, regulatory and metabolic cellular pathway and even higher-order systems. Systems biology suggests that if one gene is implicated in causing a disease or affecting a trait, other genes in the same pathway are also likely to be involved. There is evidence that this type of reasoning can improve pathway inference using combined genetic and gene-expression data [35]. This may be an important new area for research uncovering the genetic basis for personalized medicine. Research is now needed to develop al gorithms that integrate whole-genome association studies with growing knowledge of networks and pathways.

Financial \& competing interests disclosure

The author has no relevant affiliations or financial involvement with any organization or entity with a financial interest in or financial conflict with the subject matter or materials discussed in the manuscript. This indudes employment, consultancies, honoraria, stock ownership or options, expert testimony, grants or patents received or pending, or royalties

$\mathrm{No}$ writing assistance was utilized in the production of this manuscript.
Bibliography

1. Botstein D, Risch N : D iscovering genotypes underlying human phenotypes: Past successes for mendelian disease, future approaches for complex disease. $\mathrm{N}$ at. Genet. 33(Suppl.), 228-237 (2003).

2. Badano JL, Katsanis N : Beyond M endel: an evolving view of human genetic disease transmission. N at. Rev. Genet. 3(10), 779-789 (2002).

3. M acGregor AJ, Snieder H, Schork N J, Spector TD: Twins. N ovel uses to study complex traits and genetic diseases. Trends Genet. 16(3), 131-134 (2000).

4. Boomsma D, Busjahn A, Peltonen L: Classical twin studies and beyond. $N$ at. Rev. Genet. 3(11), 872-882 (2002).

5. Bader JS: The relative power of SN PS and haplotype as genetic markers for association tests. Pharmacogenomics 2(1), 11-24 (2001).

6. Giot $L$, Bader JS, Brouwer $C$ et al.: A protein interaction map of $D$ rosophila melanogaster. Science 302(5651), 1727-1736 (2003).

7. International $\mathrm{H}$ apM ap Consortium: A haplotype map of the human genome. $N$ ature 437(7063), 1299-1320 (2005).
8. W inzeler EA, Shoemaker D D, Astromoff A et al.: Functional characterization of the S. cerevisiae genome by gene deletion and parallel analysis. Science 285(5429), 901-906 (1999).

9. Krogan N J, Cagney G, Yu H et al.: G lobal landscape of protein complexes in the yeast Saccharomyces cerevisiae. N ature 440(7084), 637-643 (2006).

10. Gavin AC, Aloy P, Grandi P et al.: Proteome survey reveals modularity of the yeast cell machinery. N ature 440(7084), 631-636 (2006).

11. Jansen RC, N ap JP: G enetical genomics: The added value from segregation. Trends Genet. 17(7), 388-931 (2001).

12. Perlstein EO, Ruderfer D M , Roberts DC, Schreiber SL, Kruglyak L: G enetic basis of individual differences in the response to small-molecule drugs in yeast. $\mathrm{N}$ at. $\mathrm{G}$ enet. 39(4), 496-502 (2007).

13. Gatbonton $T$, Imbesi $M, N$ elson $M$ et al.: Telomere length as a quantitative trait: genome-wide survey and genetic mapping of telomere length-control genes in yeast. PL oS Genet. 2(3), E35 (2006).
14. N ogami S, O hya Y, Yvert G: Genetic complexity and quantitative trait loci mapping of yeast morphological traits. PLOS Genet. 3(2), E31 (2007).

15. Brem RB, Yvert G, Clinton R, Kruglyak L: $G$ enetic dissection of transcriptional regulation in budding yeast. Science 296(5568), 752-755 (2002).

16. Brem RB, Kruglyak L: The landscape of genetic complexity across 5,700 gene expression traits in yeast. Proc. N atl Acad. Sci. USA 102(5), 1572-1577 (2005).

17. Schadt EE, M onks SA, D rake TA et al.: $G$ enetics of gene expression surveyed in maize, mouse and man. N ature 422(6929), 297-302 (2003).

18. G hazalpour A, D oss S, Sheth SS et al.: $G$ enomic analysis of metabolic pathway gene expression in mice. Genome Biol. 6(7), R59 (2005).

19. Sieberts SK, Schadt EE: M oving toward a system genetics view of disease. M amm. Genome 18(6-7), 389-401 (2007).

20. Lan H, Stoehr JP, N adler ST, Schueler KL, Yandell BS, Attie AD : D imension reduction for mapping mRN A abundance as quantitative traits. Genetics 164(4), 1607-1614 (2003). 
21. Yvert G, Brem RB, Whittle J et al.: Trans-acting regulatory variation in Saccharomyces cerevisiae and the role of transcription factors. N at. Genet. 35(1), 57-64 (2003).

22. Lan $\mathrm{H}, \mathrm{Chen} \mathrm{M}$, Flowers JB et al.: Combined expression trait correlations and expression quantitative trait locus mapping PLOS G enet. 2(1), E6 (2006).

23. GuhaT hakurta D, XieT, Anand M et al.: cis-regulatory variations: a study of SN PS around genes showing cis-linkage in segregating mouse populations. BM C Genomics 235 (2006).

24. Alberts R, Terpstra P, Li Y, Breitling R, $N$ ap JP, Jansen RC: Sequence polymorphisms cause many false cis $\mathrm{QQ} T \mathrm{~L}$. PLOS ONE 2, E622 (2007).

25. D oss S, Schadt EE, D rake TA, Lusis AJ: Cis-acting expression quantitative trait loci in mice. Genome Res. 15(5), 681-691 (2005).
26. Ronald J, Brem RB, W hittle J, Kruglyak L: Local regulatory variation in Saccharomyces cerevisiae. PL OS Genet. 1(2), E25 (2005).

27. Brem RB, Storey JD, W hittle J, Kruglyak L: $G$ enetic interactions between polymorphisms that affect gene expression in yeast. $N$ ature $436(7051), 701-703$ (2005).

28. Storey JD, Akey JM , Kruglyak L: M ultiple locus linkage analysis of genomewide expression in yeast. PLOS Biol. 3(8), E267 (2005).

29. D rake TA, Schadt EE, Lusis AJ: Integrating genetic and gene expression data: Application to cardiovascular and metabolic traits in mice. M amm. Genome 17(6), 466-479 (2006).

30. Li H , Lu L, M anly KF et al.: Inferring gene transcriptional modulatory relations: A genetical genomics approach. $\mathrm{H}$ um. $\mathrm{M} \mathrm{Ol}$. Genet. 14(9), 1119-1125 (2005).
31. Bing N, H oeschele I: Genetical genomics analysis of a yeast segregant population for transcription network inference. Genetics 170(2), 533-542 (2005).

32. Schadt EE, Lamb J, Yang $X$ et al.: An integrative genomics approach to infer causal associations between gene expression and disease. N at. Genet. 37(7), 710-717 (2005).

33. Ghazal pour A, D oss S, Zhang B et al.: Integrating genetic and network analysis to characterize genes related to mouse weight. PLOS Genet. 2(8), E130 (2006).

34. Kulp D C, Jagalur $M$ : C ausal inference of regulator-target pairs by gene mapping of expression phenotypes. BM C Genomics 7 , 125 (2006).

35. Zhu J, W iener M C, Zhang $C$ et al.: Increasing the power to detect causal associations by combining genotypic and expression data in segregating populations. PLoS Comput. Biol. 3(4), E69 (2007). 